\title{
Behavioural Inhibition Effects on Responses to Threatening Speech: Differences Between Semantics and Prosody $\S$
}

\author{
Simon Busch-Moreno, Angela Gorgol, Zirong Li, Christina Ng, Vojtech Smekal, Zhenyang \\ Xi, Ash Zhang, David Vinson* \\ Division of Psychology and Language Sciences, University College London \\ *Corresponding author: d.vinson@ucl.ac.uk
}

\begin{abstract}
The present study investigates how trait anxiety affects speech processing, and whether anxiety has different processing consequences depending on speech informational properties. Participants listened to sentences in a go/no-go task where they were asked to respond to threat, which could be present in semantics, prosody, both or neither. In one experiment they were asked to attend to prosody only and ignore semantics, and in the other, attend to semantics and ignore prosody. Two additional experiments were conducted as controls, where participants responded to neutral, rather than threatening stimuli. Trait anxiety was measured psychometrically using a behavioural inhibition system scale (BIS). Results indicate that responses to threatening prosody become slightly slower and less accurate as BIS increases, while responses to threatening semantics become faster and more accurate as BIS increases. In the control experiments, responses to neutral prosody become faster and more accurate as BIS increases, but responses to neutral semantics just become less accurate as a function of BIS. We discuss these findings in terms of a combination of over-engagement with threat, threat avoidance and over-attention to threat, highlighting the need to consider that different aspects of a single stimulus may have very different consequences for anxious information processing.
\end{abstract}

Keywords: behavioural inhibition, anxiety, attention bias, threatening speech.

\footnotetext{
$\S$ Please note that the current version of this preprint has substantially changed, as we have corrected an important statistical flaw from previous versions. This has importantly altered results, hence the title change. We have also added two control experiments, making the study more robust.
} 


\section{Introduction}

The behavioural effects of threatening stimuli on anxiety have been widely studied (for reviews see: Bar-Haim et al., 2007; Cisler and Koster, 2010). Nevertheless, these approaches tend to conflate fear with anxiety and to understand threatening stimuli as a monolithic construct. Regarding the former, anxious behavioural responses to threat can be generally distinguished from fear responses, as underlying mechanisms of fear and anxiety can be dissociated (Gray and McNaughton, 2000; Robinson et al., 2019). What is less known is how different aspects of complex threatening stimuli affect anxious responses. This is relevant, because the nature of a threatening stimulus may be as relevant as the stimulation context (i.e. task), where different modalities of stimulation may influence the intensity of threat or the processing of threat. For instance, threatening language may have the potentiality of inflicting psychological harm (e.g. offensive or degrading sentences), but most often speech itself cannot directly cause physical harm. This can be considered as an indirect form of threat: language can express the potential of harm, signalling threat coming from a source that is not language itself, such as the threatening speaker themselves. This has relevant implications for approaches grouping anxiety and fear as a singular construct, and also grouping language threat and other types of threat as equivalent stimuli (Bar-Haim et al., 2007; Cisler and Koster, 2010).

Another very important consideration in understanding the relationship between threat and anxiety, is that threatening stimuli are often treated in a relatively undifferentiated manner, likely to affect anxious processing on anxiety across modality. A variety of studies have investigated threatening stimuli of varying types such as facial expressions, images of threatening objects, entities or situations, threatening sounds and threatening language (for metanalyses and reviews see: Beard et al., 2012; Chen et al., 2020; Dymond et al., 2015). Different types of stimuli can generate different effects on anxiety, such as verbal versus visual stimuli (Beard et al., 2012; Chen et al., 2020), Where language is concerned, despite the complex and distinctive informational characteristics of connected speech, linguistic stimuli tend to be treated as single/simple constructs when it comes to investigating threat (e.g. semantic stimuli, see Dymond et al., 2015). But speech can express threat simultaneously via very different informational properties, reflected in semantics and prosody. While semantics can convey meaning through lexical items within sentences, prosody can convey threat through variations in acoustic 
features of sentences, such as slow-rate spectral changes, intensity and others (Kotz and Paulmann, 2011; Paulmann and Kotz, 2008) that may affect listeners in very different ways. Some studies have researched differences between emotional semantics and prosody (e.g. Grimshaw et al., 2003; Wambacq and Jerger, 2004), but there has been very little study of threat or anxious processing from this perspective. Although more recently, some approaches have explored the differences between prosody and semantics in the context of anxiety (Pell et al., 2015). In the present study, we want to directly investigate whether prosody or semantics affect trait anxiety differently. The behavioural inhibition system (BIS) has been postulated to be a good proxy of anxiety when overactive (McNaughton and Corr, 2004), aligning with the notion of anxiety as dissociated with fear. Our main question focuses on whether the relationship between trait anxiety (i.e. BIS) and language is different when stimuli (threatening sentences) contain semantic or prosodic threat.

Previous evidence shows that anxiety can be related to both hyper vigilance to threat (fast responses) or with delayed disengagement from threat (slow responses), probably due to task-dependent effects (Cisler and Koster, 2010). While some tasks emphasise directly threatening/harming and fast stimuli, others emphasise slow and less directly threatening stimuli, indicating hypervigilance (over-attention) or delayed disengagement (over-engagement) respectively. This difference may arise from tasks tapping into different processes. The fear systems, as opposed to behavioural inhibition system (BIS), may induce faster reactions (fight, flee or freeze) to directly threatening stimuli (McNaughton and Corr, 2004); emphasising the difference between fear and anxiety (Robinson et al., 2019). Also, anxiety induced arousal (Heller et al., 1997; McNaughton and Gray, 2000) has been associated with over-attention to threat, as opposed to anxious apprehension, associated with over-engagement with threat (Spielberg et al., 2013). In short, fear systems and anxious arousal should facilitate threat detection (over-attention), while excessive BIS and anxiety-induced apprehension should interfere with disengagement from threat (over-engagement). Because prosody and semantics privilege different forms of brain processing at different processing stages (Kotz and Paulmann, 2011), it could be expected that anxiety does not affect the processing of threatening prosody or semantics evenly. This could imply, for instance, that retrieving lexical meaning from a threatening sentence could privilege faster detection of salient stimuli, as the offensive/harming lexical item expressing threat may 
change location within a sentence and needs to be quickly detected. Differently, the continuous presence of prosodic threat across most of the sentence may induce difficulty in disengaging from threat, because the exposure starts earlier and lasts for a longer time. This is consistent with a notion of anxiety inducing over-attention to threat in early processing stages (Bar-Haim et al., 2007), in particular for fast/short stimuli or timepressured tasks (Cisler and Koster, 2010). But for sentences that are threatening both semantically and prosodically, it can be very difficult to disentangle the relative contributions of these different informational properties, and the mechanisms by which they might affect anxious processing.

As the distinction between over-attention and over-engagement seems especially relevant when potentially separable effects of prosodic and semantic threat are concerned, the go/no-go paradigm can be especially useful. A typical finding is that anxiety-related response inhibition is characterised by better no-go accuracy and not reaction time increase (Grillon et al., 2017; Robinson et al., 2013). These studies used short neutral stimuli (symbols) paired with electrical shocks (threat condition) to induce anxiety. Yet, it has been pointed out that short and intense stimuli tend to favour more vigilance (over-attention) to threat, rather than difficulty in disengaging (overengagement) from threat, the latter favoured by longer and less directly threatening stimuli (Cisler and Koster, 2010). Indeed, recent studies indicate that in a spatial cue task, over-engagement with threatening sounds occurs only when anxious participants have enough time to engage with threat, and is associated with increased RTs (Wang et al., 2019). BIS models would predict that during fast presentation of strongly aversive stimuli in a go/no-go task, anxiety should improve no-go accuracy (inhibition) without altering reaction times (Grillon et al., 2017). This may be related to increased arousal during risk assessment in preparation for fear (e.g. flight) responses (Gray and McNaughton, 2000). Nevertheless, longer periods of exposure to milder potential threat, thus keeping BIS engaged for longer times, could also induce internal scanning (e.g. risk assessment through memory), resulting in slower processing (Gray and McNaughton, 2000).

This difference between anxiety-induced arousal or apprehension (Heller, 1997) should not be reflected on task variations, but should depend on three factors being present in the task: 1) The indirectness of threatening stimuli, such as language/speech, which can imply potential harm but cannot be strongly harmful on its own. 2) Longer 
exposure to potential or implied threat, such as longer inter stimulus intervals (ISI), longer speech stimuli (i.e. sentences), or both. 3) Participants' predisposition to worry or BIS, which should provide the main association between anxious apprehensive traits (trait anxiety) and slower processing as reflected by reaction times (RTs) slowing down for longer stimuli or in tasks where reflection before responding is possible. If this is true, we expect that the effects of BIS (trait anxiety, apprehension) should imply a sizable slowdown in reaction times (RTs) as well as improved accuracy, a speed-accuracy trade-off as shown in previous go/no-go literature (Grillon et al., 2017; Robinson et al., 2013).

In the present study we investigate whether anxious people will exhibit overengagement with threat in go/no-go tasks involving detection of threat in acoustically presented sentences. We operationalised anxiety using the BIS scale from the Reinforcement Sensitivity Theory Personality Questionnaire (RST-PQ) (Corr and Cooper, 2016). This measure is well grounded in the physiological understanding of BIS and its relationship to anxiety (e.g. McNaughton and Corr, 2004). Under this approach, BIS can be understood as a proxy of anxiety; namely, higher levels of BIS should be indicative of higher trait anxiety. Crucially, in order to tease apart the different contributions of semantic and prosodic threat, we factorially manipulated these dimensions in creating materials: threatening in both dimensions (Congruent threat), threatening prosody and neutral semantics (Prosodic threat), threatening semantics and neutral prosody (Semantic threat), and neutral in both dimensions (no threat). Furthermore, in separate web-based experiments using these same materials we asked participants to attend to only one of the two dimensions in making a "go" response. In Experiment 1 (Prosody Experiment) participants answer to acoustic threat and ignore threatening semantic content, while in Experiment 2 (Semantic Experiment) participants answer to threatening semantic content and ignore acoustics.

From the perspective of phasic models of anxiety (i.e. Bar-Haim et al., 2007; Cisler and Koster, 2010), increases in anxiety will lead to increases in accuracy, possibly accompanied by anxiety-associated slowdowns for experiments 1 and 2; if the longer stimulus duration permits further engagement with threat. Moreover, we should not expect different patterns of results for prosodic vs semantic threat detection despite their differences in informational properties; if speech can indeed be understood as a monolithic stimulus (i.e. informational properties are irrelevant for threat induction). The extent to which conflicting cues affect responses (i.e. Congruent: threatening in both 
semantics and prosody, vs conditions in which threat is conveyed in only one of the two) will also allow us to explore the influence of the task-irrelevant cue.

As recommended by anonymous reviewers, we also carried out two control experiments, using the same materials but instead asking participants to respond only to stimuli that were neutral, rather than threatening, on a given dimension. In Experiment 3 , make a "go" response when prosody was neutral, withhold response to threatening prosody; in Experiment 4: make a "go" response when semantics was neutral, withhold responses to threatening semantics. This enabled us to test whether effects of anxiety observed in Experiments 1 and 2 could be attributed directly to threat in the stimulus presented, or might be more general to the types of tasks employed (e.g. are anxious people more accurate/ slower in these types of tasks). Based on the previous anxiety literature we would predict that no effects of BIS should be observed in Experiments 3 and 4, as "go" responses should only be made to stimuli that are neutral in the relevant dimension. However, it may also be that anxious people are sensitive to threat regardless of whether it is task-relevant, in which case BIS should not affect responses to Neutral stimuli but should still have effects on Semantic or Prosodic stimuli, that depict threat in the unattended dimension. Finally, if anxiety has even more general effects on tasks like these, effects of anxiety when responding to neutral stimuli (Experiments 3 and 4) should be comparable to those when responding to threat (Experiments 1 and 2).

\section{Methods}

\section{Participants}

Participants for Experiment 1 ( $\mathrm{n}=40$; age: mean $=33.45, \mathrm{SD}=9.09 ; 29$ females), Experiment 2 ( $\mathrm{n}=49$; age: mean $=36.14, \mathrm{SD}=10.52 ; 26$ females), Experiment 3 ( $\mathrm{n}=38$; age: mean $=32.76, \mathrm{SD}=9.97 ; 27$ Females $)$, and Experiment $4(\mathrm{n}=46$; age: mean $=35.54$, $\mathrm{SD}=13.30 ; 29$ Females) were recruited using Prolific and completed the study on Gorilla (gorilla.sc). They reported not having hearing, psychological or neurological problems; having English as their first language; and being right-handed. Participants were compensated at a $£ 7.50 /$ hour rate. All the procedure counts with UCL's ethical approval and participants' data was handled according to GDPR protocol as informed to them. Participants were excluded if they gave zero responses to either target category or answered to the opposite category. Experiment 1: 10 participants gave more responses 
to Semantic than Prosody and were excluded. Experiment 2: 1 participant gave more responses to Prosody than Semantic and was excluded. Experiment 3: 4 participants gave more responses to Prosody than Semantic (neutral prosody) and were excluded. Experiment 4: 12 participants gave more responses to Semantic than Prosody (neutral semantic) and were excluded.

\section{Materials}

Sentences (52 per category) were created as follows. Sentences were recorded in an isolated anechoic chamber by a male native English speaker (amateur actor) using a RODE NT1-A1 microphone. Stimuli categories include: Prosody (only prosodic threat) and Semantic (only semantic threat), Congruent (both types of threat), and Neutral (no threat). Table 1 summarises sentences' average number of words and duration. Sentences' acoustic measures were extracted using Praat via Python Parselmouth (Jadoul et al., 2018), and included Median Pitch (median of whole sentence's fundamental frequency), and Hammarberg Index (difference between 0-2000 and 2000-5000 frequency range), measuring pitch and voice quality (roughness) respectively. Sentences' lexical norms were based on one offensive word contained in each sentence and included average Arousal and Valence scores from the extended Affective Norms for English Words (Warriner et al., 2013). Figure 1 shows oscillograms and spectrograms of example sentences on each category. Table 2 shows Bayesian estimation supersedes the t-test (BEST) (Kruschke, 2013) estimated means and SDs, and Figure 2 shows BEST differences between means.

Table 1. Sentences' average number of words and duration

\begin{tabular}{lcccc}
\hline Type & \#Words Mean & \#Words SD & Duration Mean & Duration SD \\
\hline Congruent & 5.16 & 1.03 & 1519.08 & 233.67 \\
\hline Neutral & 4.61 & 1.03 & 1471.37 & 257.65 \\
\hline Prosody & 5.02 & 0.73 & 1509.69 & 172.19 \\
\hline Semantic & 5.16 & 1.01 & 1518.20 & 215.96 \\
\hline
\end{tabular}



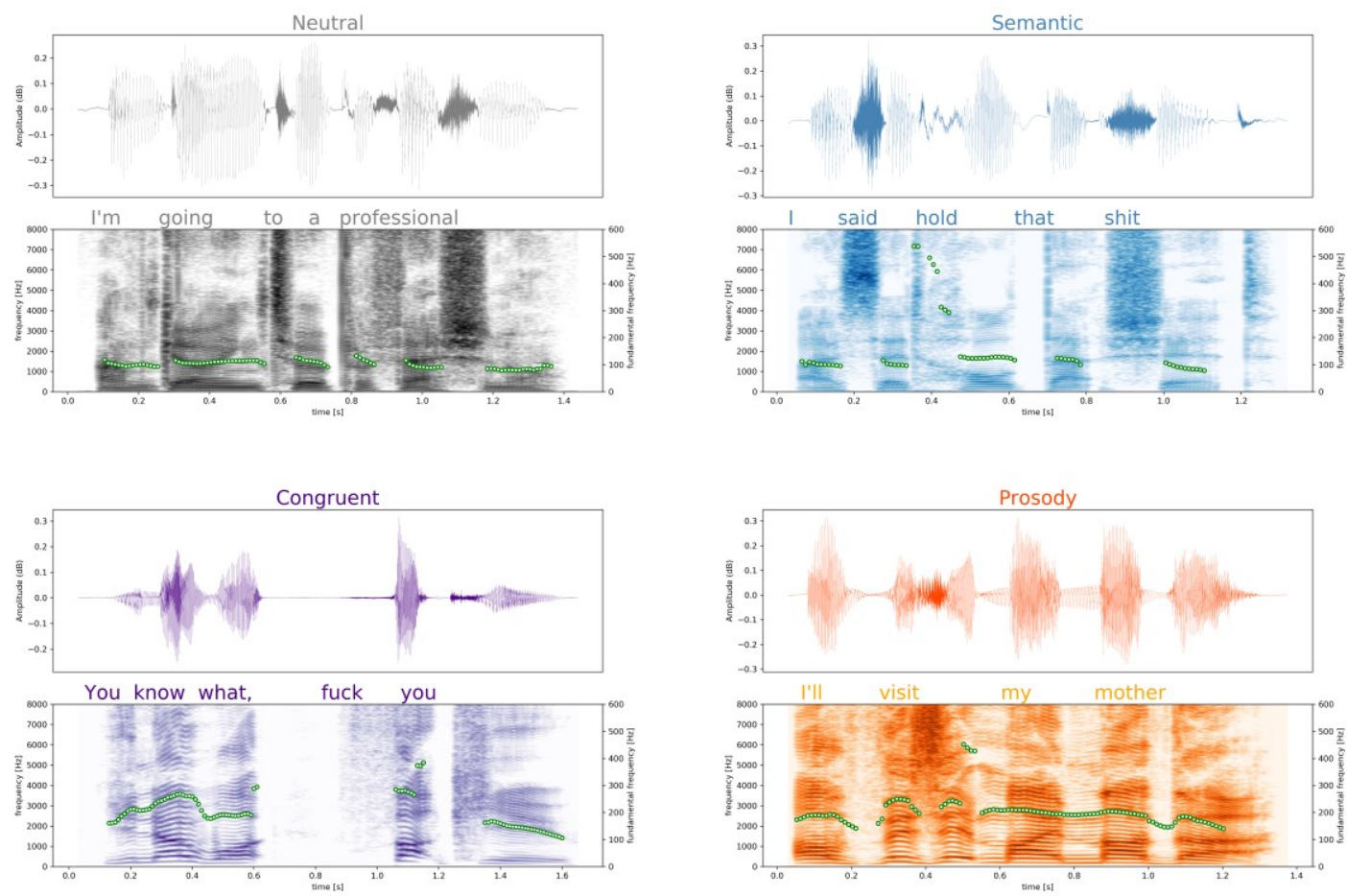

Figure 1. Example of four sentences used in this study. Top of each image: oscillogram showing amplitude changes. Bottom of each image: spectrogram showing frequency changes. Top left: neutral prosody and neutral semantics (Neutral). Top right: threatening prosody and threatening semantics (Congruent). Bottom left: neutral prosody and threatening semantics (Semantic). Bottom right: threatening prosody and neutral semantics (Prosody). Green dots indicate fundamental frequency (F0) contours.

Table 2. BEST estimated average posterior distributions for Mean and SD

\begin{tabular}{lcccccccc}
\hline Measure & \multicolumn{2}{c}{ Arousal } & \multicolumn{2}{c}{ Valence } & \multicolumn{2}{c}{ Median Pitch } & \multicolumn{2}{c}{ Hammarberg Index } \\
\hline Category & Mean & SD & Mean & SD & Mean & SD & Mean & SD \\
\hline Neutral & 3.96 & 0.67 & 5.94 & 0.91 & 109.17 & 4.74 & 22.74 & 4.13 \\
\hline Congruent & 5.96 & 0.89 & 3.49 & 1.26 & 197.89 & 10.96 & 19.66 & 3.50 \\
\hline Prosody & 4.04 & 0.69 & 6.17 & 0.88 & 200.17 & 11.29 & 20.38 & 3.88 \\
\hline Semantic & 5.94 & 0.86 & 3.44 & 1.23 & 108.99 & 4.57 & 23.00 & 3.60 \\
\hline
\end{tabular}

\section{Procedure}

Tasks were web-based and were presented in Gorilla (gorilla.sc). In all experiments, after signing consent and confirmation of requirements, participants completed the BIS scale questionnaire and then proceeded to a brief practice task (10 stimuli) before proceeding to the main task. Experiment 1: participants had to answer to 
Congruent and Prosody (threatening sound) by pressing the space bar on their keyboards as fast as possible and before the sentence ended but withhold responses to Semantic and Neutral. Experiment 2: participants had to answer to Congruent and Semantic (threatening content) by pressing the space bar on their keyboards as fast as possible and before the sentence ended but withhold responses to Prosody and Neutral. Experiment 3: participants had to answer to Neutral and Semantic (neutral prosody) by pressing the space bar on their keyboards as fast as possible and before the sentence ended but withhold responses to Prosody and Congruent. Experiment 4: participants had to answer to Neutral and Prosody (neutral content) by pressing the space bar on their keyboards as fast as possible and before the sentence ended but withhold responses to Semantic and Congruent. Stimuli were presented randomly with a $1.5 \mathrm{~s}$ inter stimulus interval (ISI) between them.

\section{Analysis}

Before analysis, all responses that were made in the first $300 \mathrm{~ms}$ after sentence end were coded as hits or false alarms (FAs) depending on correct or incorrect preceding trial respectively, the rest were kept as misses or correct rejections (CR). For accuracy analyses, a logistic regression was implemented. This model uses varying intercepts and slopes over each sentence type (Neutral, Congruent, Prosody, Semantic), which is input as a matrix operation with the parameter $(\alpha \cdot A, \beta \cdot B)$. This linear model is input into an inverse logit function. Observations correspond to binary values of response (response = 1 , no-response $=0$ ), which will allow to determine the probability of participants answering to any of the four categories; that is, the probability of Hits and FAs. For the Prosody experiment, Hits correspond to Prosody and Congruent sentences, and FAs to Semantic and Neutral sentences. For the Semantic experiment, Hits correspond to Semantic and Congruent sentences, and FAs to Prosody and Neutral sentences. Figure 3 summarises this model. For RT analyses, a robust hierarchical regression was implemented. We initially considered incorporating subject varying intercepts but excluded them from the present analyses due to convergence problems and possible collinearity between BIS and subject variables. Figure 4 summarises this model. 

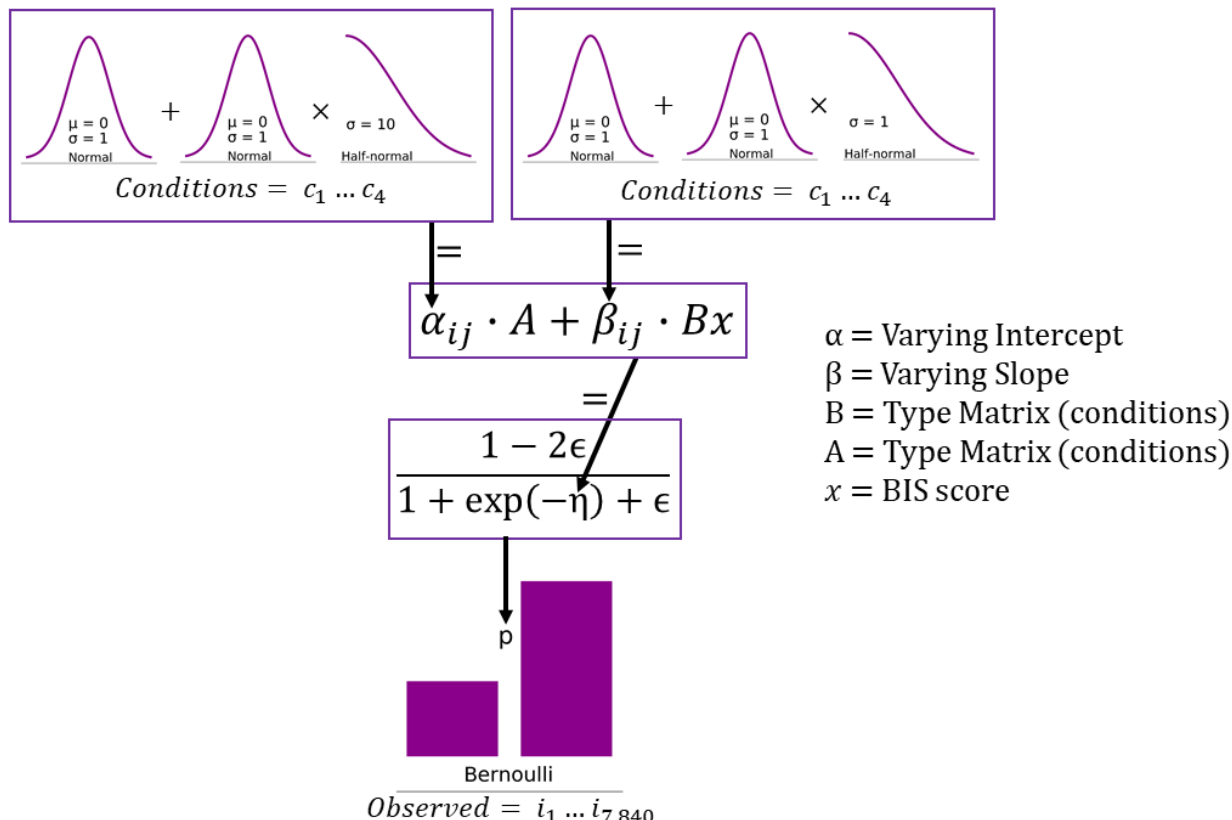

Observed $=i_{1} \ldots i_{7,840}$

Figure 2. Diagram representation of hierarchical logistic regression model. Arrows indicate the relationship between a parameter and priors or hyperpriors, where tilde $(\sim)$ indicates a stochastic relationship and equal $(=)$ indicates a deterministic relationship. Type matrices correspond to design matrices containing the four sentence types. Observations correspond to responses/no-responses given to a sentence at given trial (0-1 Bernoulli trials) (Experiment 1).

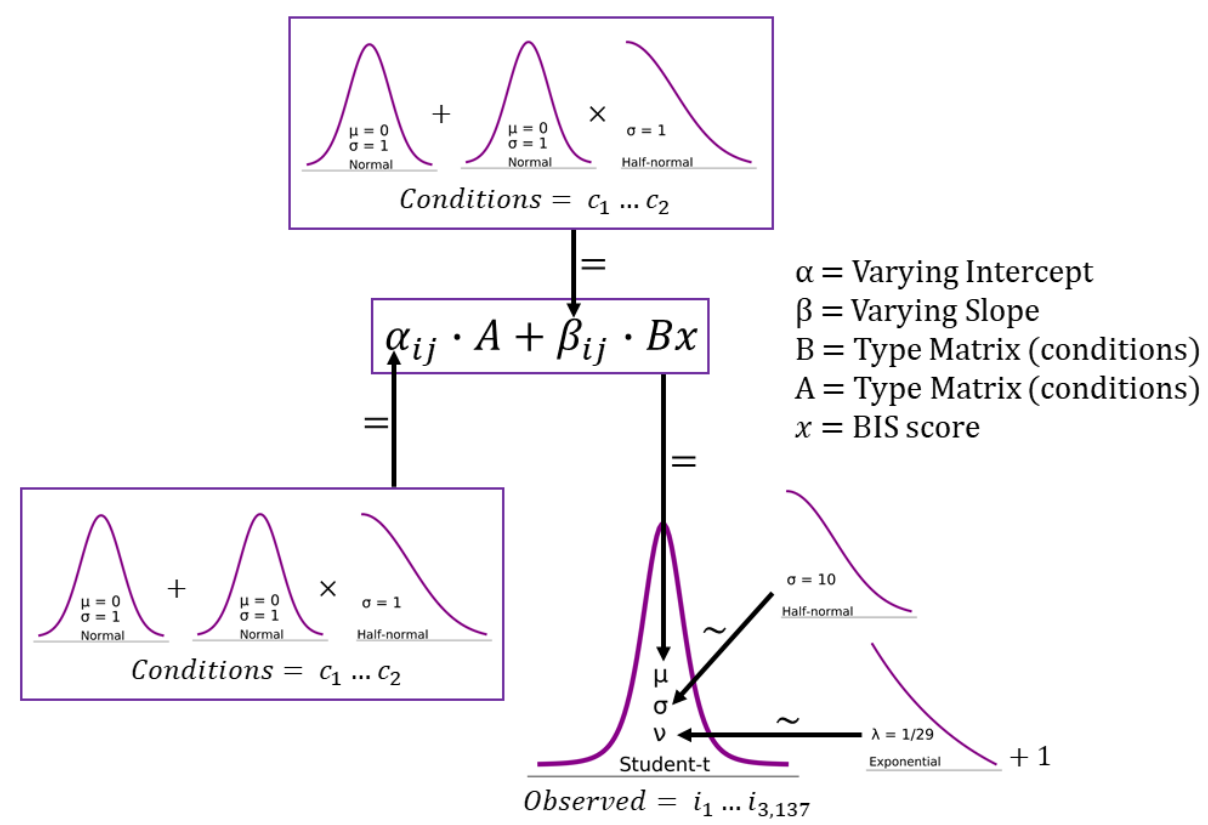

Figure 3. Diagram representation of hierarchical robust regression model. Arrows indicate the relationship between a parameter and priors or hyperpriors, where tilde $(\sim)$ indicates a stochastic relationship and equal $(=)$ indicates a deterministic relationship. Type matrices correspond to design matrices containing the two target sentence types (e.g. Congruent and Prosody). Observations correspond to reaction times (RTs) in milliseconds (Experiment 1). 
Both accuracy and RT models (Figures 3 and 4) were implemented in the same manner for all experiments, where number of observations and participants changed accordingly. Note that given our previous experiment's generally good precision of estimates, we consider that similar sample sizes (e.g. near 40 to 50 participants) is appropriate for present purposes. Also note that the rationale behind accuracy models is having information about responses to each type via estimates obtained through sentence type matrices, thus having information about responses to targets and distractors. In terms of signal detection theory these can be understood as signal and noise (Hit/FA, CR/Miss), where estimated log-odds will inform about sensitivity and bias. The RT models follow a robust regression to give a better account of outlying observations. Models were sampled via the Markov Chain Monte Carlo (MCMC) No-u Turn Sampler (NUTS) method provided by PyMC3 (Salvatier et al., 2016), using 4 chains, 2000 tuning samples, and 2000 samples each. Results were assessed by using estimate increase and HDIs from expected values. Control experiments, Experiment 3 and Experiment 4 were designed, sampled and assessed in the same manner.

\section{Results}

\section{Experiment 1 (Detect threatening prosody)}

Models converged well (all $\hat{R} s \leq 1.01, E S S>400, B F M I s>0.7$ ). For detailed summaries and additional plots see our OSF repository (https://osf.io/ptcr9/). Accuracy results indicate that most participants were able to discriminate signal and noise efficiently. Note that response corresponds to any type of response, which was input in the model as a Bernoulli trial ( $0=$ no-response, $1=$ response), where response can be Hits and False Alarms (FAs) and no-responses are Misses and correct rejections (CRs). So, for Experiment 1, the computed probability is for Hits (Prosody and Congruent) and False Alarms (Semantic and Neutral). Table 3 and Figure 5 summarise these results. These generally indicate that the probability to answering to Congruent slightly decreases around $7 \%$ as a function of BIS (from near $88 \%$ to around $81 \%$ ), while it remains generally constant for Prosody (around 75\%). The probability of answering Neutral is less than $1 \%$ and to Semantic is between $2 \%$ to $3 \%$ with greater uncertainty (here and elsewhere, we use the term "uncertainty" to refer to uncertainty of the estimates, that result in broader, and possibly overlapping HDIs). 
Table 3. Experiment 1 accuracy estimates

\begin{tabular}{cccccc}
\hline BIS & Sentence Type & Mean & SD & HDI 5\% & HDI 95\% \\
\hline 1 & Neutral & 0.61 & 0.30 & 0.18 & 1.01 \\
\hline 1 & Prosody & 75.58 & 2.11 & 72.10 & 79.02 \\
\hline 1 & Semantic & 3.53 & 0.81 & 2.23 & 4.77 \\
\hline 1 & Congruent & 87.72 & 1.51 & 85.31 & 90.22 \\
\hline 62 & Neutral & 0.37 & 0.18 & 0.09 & 0.63 \\
\hline 62 & Prosody & 75.56 & 1.98 & 72.32 & 78.80 \\
\hline 62 & Semantic & 2.35 & 0.56 & 1.42 & 3.23 \\
\hline 62 & Congruent & 80.82 & 1.89 & 77.81 & 84.00 \\
\hline
\end{tabular}

Note. Estimates are probabilities (in \%) from expectation's posterior (intercept plus slope). 

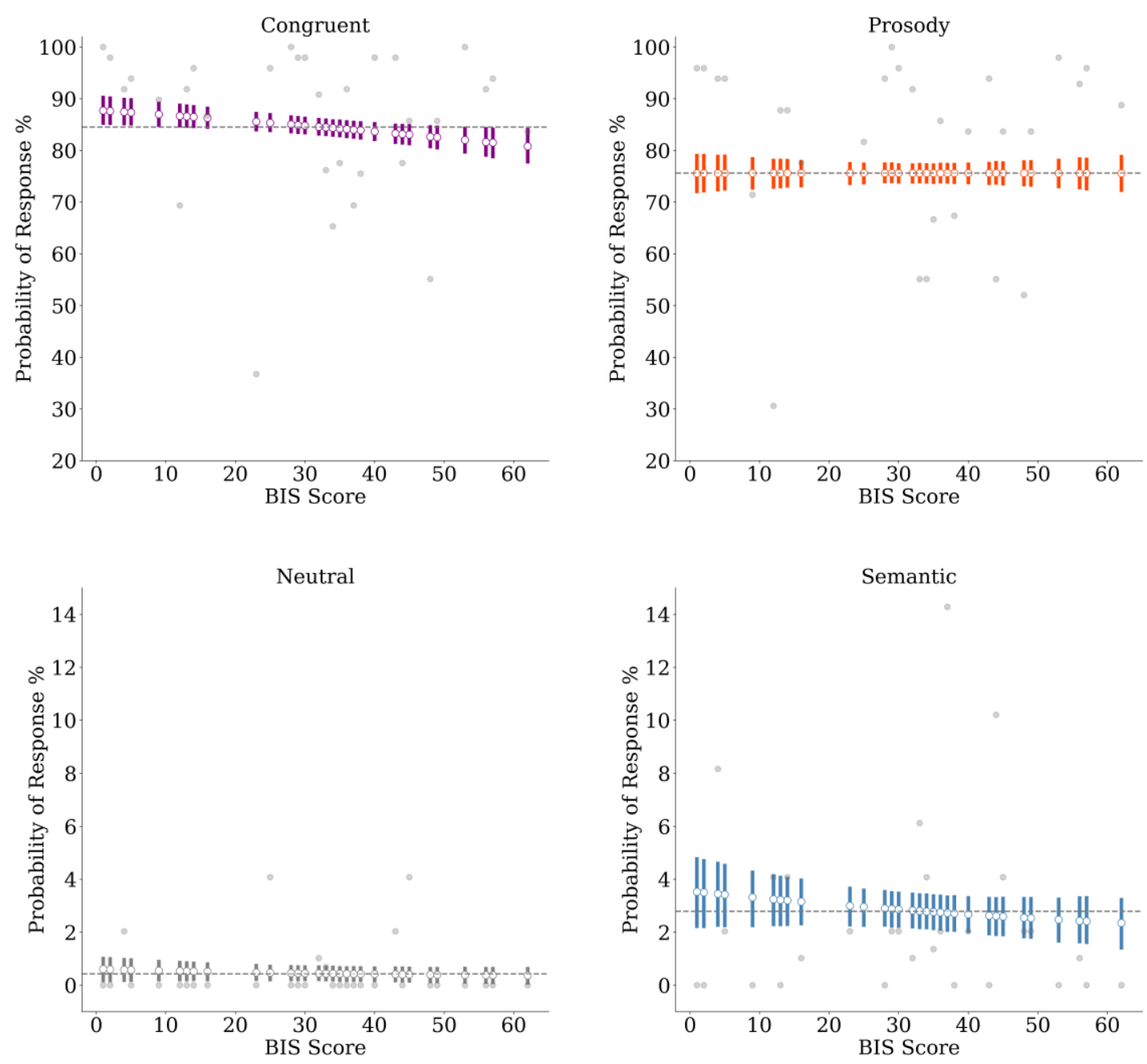

Figure 4. Experiment 1 accuracy estimates. Plots show responses to stimuli containing threatening prosody. Circles represent posterior means; bars indicate highest density intervals (HDIs). Grey dots indicate raw means by BIS score. Grey dashed lines indicate estimated posterior median accuracy by median BIS per condition.

Results from RT robust regression indicate that as BIS increases RTs slightly increase (slower responses). RTs to Prosody increase around 65ms from lowest to highest BIS score, while RTs to Congruent increase around 98ms. Note that estimations for Prosody almost present HDI overlap, but estimations for Congruent indicate better certainty. See Table 4 and Figure 6 for summaries of these results. 
Table 4. Experiment 1 reaction time estimates

\begin{tabular}{cccccc}
\hline BIS & Sentence Type & Mean & SD & HDI 5\% & HDI 95\% \\
\hline 1 & Prosody & 1103.42 & 19.48 & 1070.64 & 1134.77 \\
\hline 1 & Congruent & 1115.40 & 18.62 & 1083.64 & 1144.32 \\
\hline 62 & Prosody & 1168.51 & 18.02 & 1138.85 & 1197.83 \\
\hline 62 & Congruent & 1213.36 & 18.15 & 1181.85 & 1241.53 \\
\hline
\end{tabular}

Note. Estimates are reaction times (in milliseconds) from expectation's posterior (intercept plus slope).

Threatening Prosody Regressions
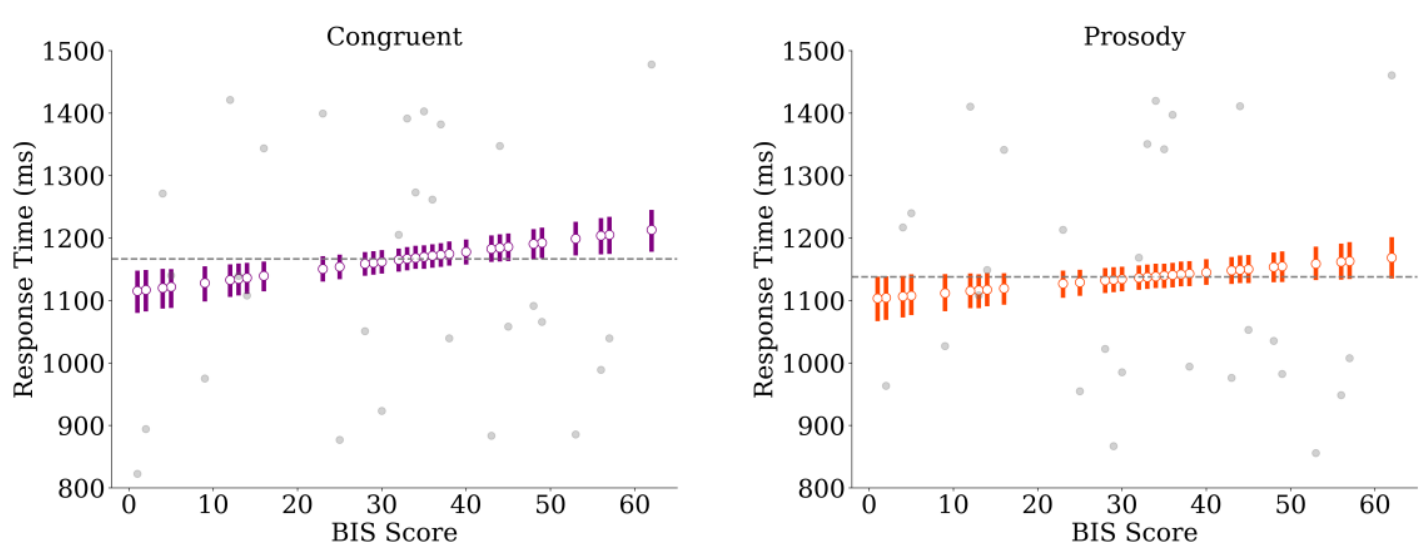

Figure 5. Experiment 1 reaction time estimates. Plots show responses to stimuli containing threatening prosody. Circles represent posterior means; bars indicate highest density intervals (HDIs). Grey dots indicate raw means by BIS score. Grey dashed lines indicate estimated posterior median accuracy by median BIS per condition.

In summary, Experiment 1 results indicate that accuracy is generally high for both Congruent and Prosody sentence types with medians around $85 \%$ and $75 \%$ respectively. However, estimated accuracy barely changes as a function of BIS (only a minimal decrease for Congruent), suggesting negligible effects of BIS. Median RT remains around $1100 \mathrm{~ms}$ for both sentence types. RTs slightly increase as a function of BIS, with a slightly more certain increase for Congruent, suggesting small effects of BIS. 
Experiment 2 (Detect threatening semantics)

Models converged well (all $\hat{R} s \leq 1.01$, ESS $>600$, BFMIs $>0.7$ ). For detailed summaries and additional plots see our OSF repository (https://osf.io/ptcr9/). Accuracy results indicate that most participants were able to discriminate signal and noise efficiently. Note that response corresponds to any type of response, which was input in the model as a Bernoulli trial ( $0=$ no-response, $1=$ response $)$, where response can be Hits and False Alarms (FAs) and no-responses are Misses and correct rejections (CRs). So, for Experiment 2, the computed probability is for Hits (Semantic and Congruent) and False Alarms (Prosody and Neutral). Table 5 and Figure 7 summarise these results. These generally indicate that the probability to answering to Congruent increases around $17 \%$ as a function of BIS (from 59\% to 76\%), and increases near 15\% for Semantic (from 57\% to $72 \%$ ). The probability of answering Neutral is less than $1 \%$, but Prosody shows a small increase of around 3\% from lowest to highest BIS score (wide HDIs but not overlapping).

Table 5. Experiment 2 accuracy estimates

\begin{tabular}{cccccc}
\hline BIS & Sentence Type & Mean & SD & HDI 5\% & HDI 95\% \\
\hline 2 & Neutral & 0.42 & 0.21 & 0.16 & 0.67 \\
\hline 2 & Prosody & 2.79 & 0.48 & 2.00 & 3.55 \\
\hline 2 & Semantic & 57.07 & 2.08 & 53.60 & 60.39 \\
\hline 2 & Congruent & 59.15 & 2.06 & 55.77 & 62.51 \\
\hline 63 & Neutral & 0.67 & 0.29 & 0.19 & 1.10 \\
\hline 63 & Prosody & 5.74 & 1.09 & 4.01 & 7.42 \\
\hline 63 & Semantic & 72.24 & 2.23 & 68.77 & 76.05 \\
\hline 63 & Congruent & 76.31 & 2.03 & 72.95 & 79.73 \\
\hline
\end{tabular}

Note. Estimates are probabilities (in \%) from expectation's posterior (intercept plus slope). 

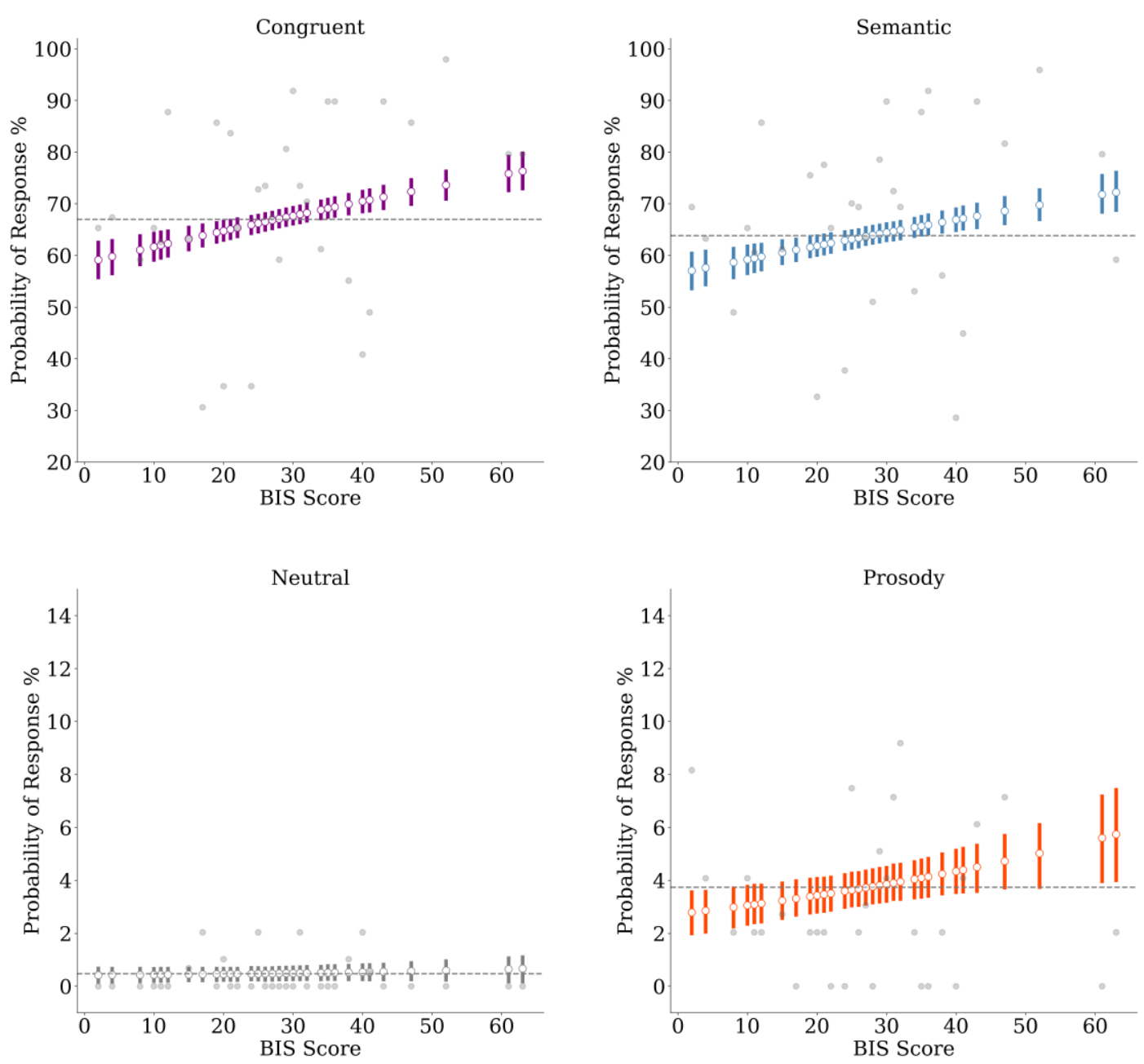

Figure 6. Experiment 2 accuracy estimates. Plots show responses to stimuli containing threatening semantics. Circles represent posterior means; bars indicate highest density intervals (HDIs). Grey dots indicate raw means by BIS score. Grey dashed lines indicate estimated posterior median accuracy by median BIS per condition.

Robust regression results indicate that as BIS increases RTs decrease (faster responses). RTs to Semantic increase around 91ms from lowest to highest BIS score, while RTs to Congruent increase around 144ms. Responses to Congruent are, overall, between $42 \mathrm{~ms}$ and $95 \mathrm{~ms}$ faster respect to Semantic. Note that estimations indicate precise HDIs not close to overlap, in particular for Congruent. See Table 6 and Figure 8 for summaries of these results. 
Table 6. Experiment 2 reaction time estimates

\begin{tabular}{cccccc}
\hline BIS & Sentence Type & Mean & SD & HDI 5\% & HDI 95\% \\
\hline 2 & Semantic & 1652.76 & 17.92 & 1625.85 & 1684.31 \\
\hline 2 & Congruent & 1610.30 & 17.50 & 1580.33 & 1637.12 \\
\hline 63 & Semantic & 1561.32 & 21.93 & 1526.45 & 1598.20 \\
\hline 63 & Congruent & 1466.02 & 20.75 & 1432.77 & 1500.73 \\
\hline
\end{tabular}

Note. Estimates are reaction times (in milliseconds) from expectation's posterior (intercept plus slope).

Threatening Semantic Regressions
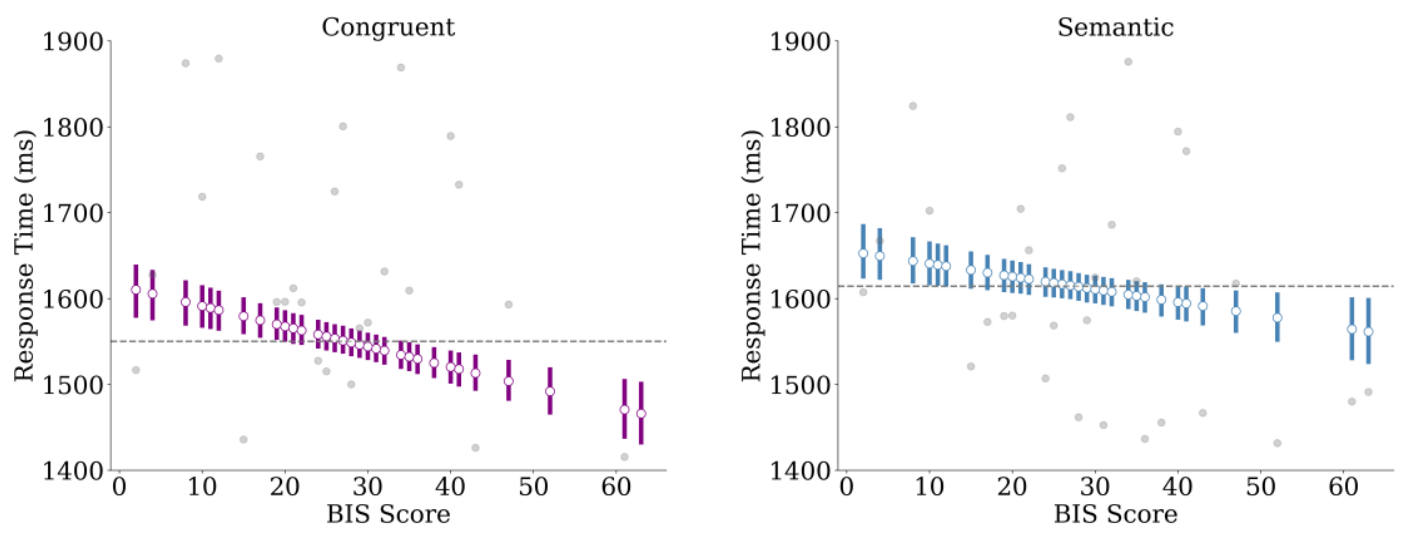

Figure 7. Experiment 2 reaction time estimates. Plots show responses to stimuli containing threatening semantics. Circles represent posterior means; bars indicate highest density intervals (HDIs). Grey dots indicate raw means by BIS score. Grey dashed lines indicate estimated posterior median accuracy by median BIS per condition.

In summary, Experiment 2 results indicate that accuracy is similar for both Congruent and Semantic sentence types with medians around $67 \%$ and $65 \%$ respectively, which also indicates relatively low accuracy overall. However, estimated accuracy shows a sustained increase as a function of BIS for both types, suggesting relevant effects of BIS. Median RTs approach 1600ms for both types, substantially longer than in Experiment 1, but are around $51 \mathrm{~ms}$ higher for Semantic than for Congruent. RTs decrease as a function of BIS, with a slightly more certain decrease for Congruent, suggesting relatively relevant effects of BIS. 


\section{Experiment 3 (Detect neutral prosody)}

Models converged well (all $\hat{R} s \leq 1.01$, ESS $>500$, BFMIs $>0.6$ ). For detailed summaries and additional plots see our OSF repository (https://osf.io/ptcr9/). Accuracy results indicate that most participants were able to discriminate signal and noise efficiently. Note that response corresponds to any type of response, which was input in the model as a Bernoulli trial ( $0=$ no-response, $1=$ response), where response can be Hits and False Alarms (FAs) and no-responses are Misses and correct rejections (CRs). So, for Experiment 3, the computed probability is for Hits (neutral Prosody and Neutral) and False Alarms (neutral Semantic and Congruent). Table 7 and Figure 9 summarise these results. These generally indicate that the probability to answering to Neutral increases around $30 \%$ as a function of BIS (from 69\% to 91\%), and increases around $43 \%$ for neutral Prosody (from 51\% to 94\%). The probability of answering Congruent remains generally constant around 3-5\%, and neutral Semantic shows a similar pattern around 3$6 \%$. Note that the great increases of Neutral and neutral Prosody may be modulated by few outliers, so accuracy increases as a function of BIS may be less extreme.

Table 7. Experiment 3 accuracy estimates

\begin{tabular}{cccccc}
\hline BIS & Sentence Type & Mean & SD & HDI 5\% & HDI 95\% \\
\hline 3 & Neutral & 69.47 & 2.82 & 64.70 & 73.95 \\
\hline 3 & Semantic (neutral) & 5.89 & 1.34 & 3.68 & 7.97 \\
\hline 3 & Prosody (neutral) & 51.17 & 3.13 & 46.10 & 56.34 \\
\hline 3 & Congruent & 5.05 & 1.25 & 3.02 & 6.98 \\
\hline 62 & Neutral & 91.29 & 1.38 & 89.16 & 93.63 \\
\hline 62 & Semantic (neutral) & 3.51 & 1.00 & 1.84 & 4.99 \\
\hline 62 & Prosody (neutral) & 93.94 & 1.01 & 92.30 & 95.61 \\
\hline 62 & Congruent & 3.53 & 1.02 & 1.92 & 5.11 \\
\hline
\end{tabular}

Note. Estimates are probabilities (in \%) from expectation's posterior (intercept plus slope). 

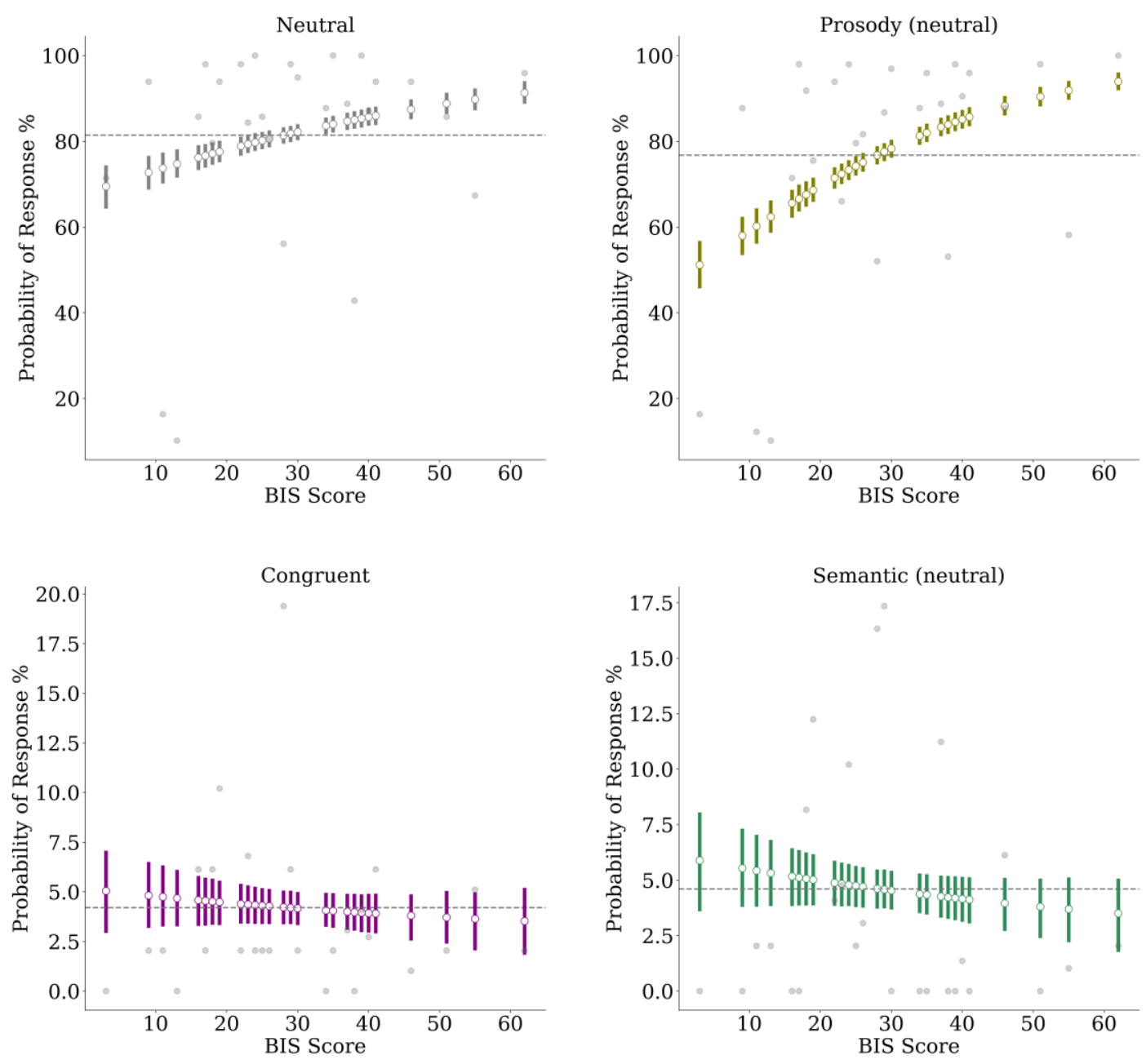

Figure 8. Experiment 3 accuracy estimates. Plots show responses to stimuli containing neutral prosody. Circles represent posterior means; bars indicate highest density intervals (HDIs). Grey dots indicate raw means by BIS score. Grey dashed lines indicate estimated posterior median accuracy by median BIS per condition.

Robust regression results indicate that as BIS increases RTs decrease (faster responses). RTs to neutral Prosody decrease around 798ms from lowest to highest BIS score, while RTs to Neutral increase around $744 \mathrm{~ms}$. Note that estimations indicate precise HDIs not close to overlap. Although a robust regression was performed to account for outliers, these extreme RT decreases need to be interpreted cautiously. See Table 6 and Figure 8 for summaries of these results. 
Table 8. Experiment 3 reaction time estimates

\begin{tabular}{cccccc}
\hline BIS & Sentence Type & Mean & SD & HDI 5\% & HDI 95\% \\
\hline 3 & Neutral & 1577.55 & 22.44 & 1537.92 & 1611.65 \\
\hline 3 & Prosody (neutral) & 1645.66 & 28.01 & 1601.36 & 1692.58 \\
\hline 62 & Neutral & 901.29 & 24.42 & 861.25 & 941.64 \\
\hline 62 & Prosody (neutral) & 847.70 & 27.44 & 800.07 & 890.13 \\
\hline
\end{tabular}

Note. Estimates are reaction times (in milliseconds) from expectation's posterior (intercept plus slope).

Neutral Prosody Regressions
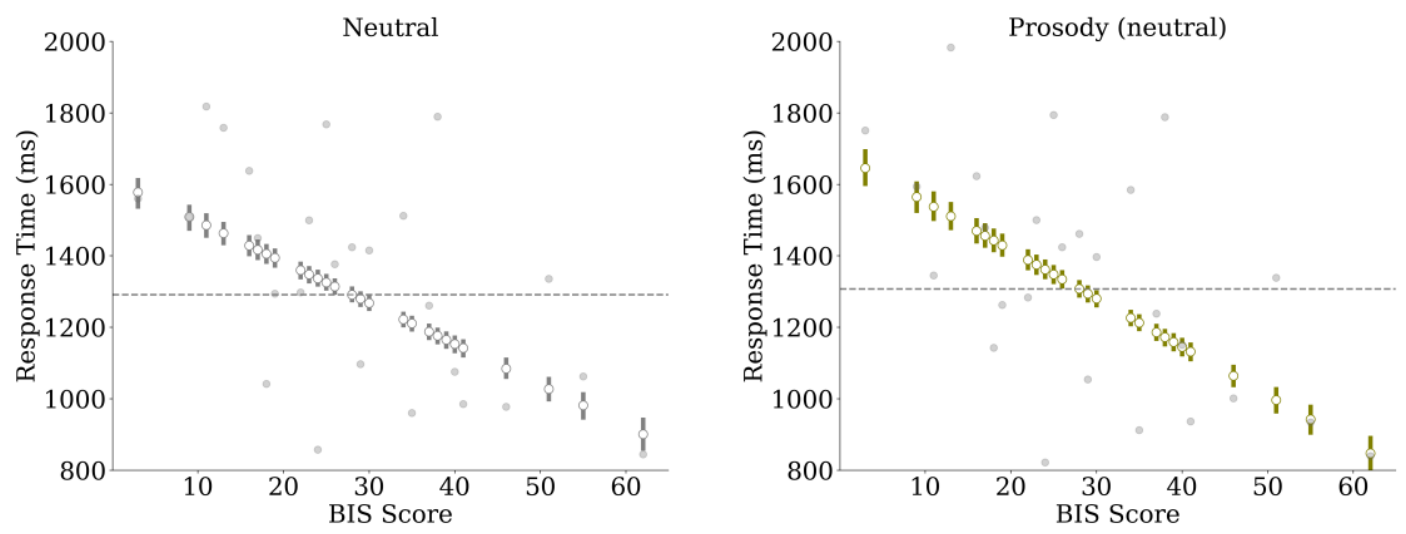

Figure 9. Experiment 3 reaction time estimates. Plots show responses to stimuli containing neutral prosody. Circles represent posterior means; bars indicate highest density intervals (HDIs). Grey dots indicate raw means by BIS score. Grey dashed lines indicate estimated posterior median accuracy by median BIS per condition.

In summary, Experiment 3 results indicate that accuracy is similarly high for both Neutral and neutral Prosody sentence types, with medians near 80\%. Estimated accuracy shows a sustained and strong increase as a function of BIS for both types, suggesting relevant effects of BIS. Median RT reaches around 1300ms for both sentence types. RTs strongly decrease as a function of BIS, suggesting relevant effects of BIS. 
Experiment 4 (Detect neutral semantics)

Models converged well (all $\hat{R} s \leq 1.01, E S S>1000, B F M I s>0.7$ ). For detailed summaries and additional plots see our OSF repository (https://osf.io/ptcr9/). Accuracy results indicate that most participants were able to discriminate signal and noise efficiently. Note that response corresponds to any type of response, which was input in the model as a Bernoulli trial ( $0=$ no-response, $1=$ response), where response can be Hits and False Alarms (FAs) and no-responses are Misses and correct rejections (CRs). So, for Experiment 4, the computed probability is for Hits (neutral Semantic and Neutral) and False Alarms (neutral Prosody and Congruent). Table 9 and Figure 10 summarise these results. These generally indicate that the probability to answering to Neutral decreases around $13 \%$ as a function of BIS (from near 69\% to around 54\%), and decreases near $21 \%$ for neutral Semantic (from $70 \%$ to $49 \%$ ). The probability of answering Congruent remains generally constant around 5-7\%, and neutral Prosody shows a similar pattern around $7 \%$.

Table 9. Experiment 4 accuracy estimates

\begin{tabular}{cccccc}
\hline BIS & Sentence Type & Mean & SD & HDI 5\% & HDI 95\% \\
\hline 5 & Neutral & 67.81 & 1.87 & 64.81 & 70.99 \\
\hline 5 & Semantic (neutral) & 70.14 & 1.89 & 66.91 & 73.10 \\
\hline 5 & Prosody (neutral) & 7.33 & 1.05 & 5.60 & 9.03 \\
\hline 5 & Congruent & 5.46 & 0.92 & 3.95 & 6.91 \\
\hline 61 & Neutral & 54.37 & 2.82 & 49.74 & 59.00 \\
\hline 61 & Semantic (neutral) & 49.31 & 2.94 & 44.74 & 54.32 \\
\hline 61 & Prosody (neutral) & 7.54 & 1.47 & 5.13 & 9.86 \\
\hline 61 & Congruent & 6.84 & 1.50 & 4.35 & 9.14 \\
\hline
\end{tabular}

Note. Estimates are probabilities (in \%) from expectation's posterior (intercept plus slope). 
Neutral Semantic Logistic Regressions
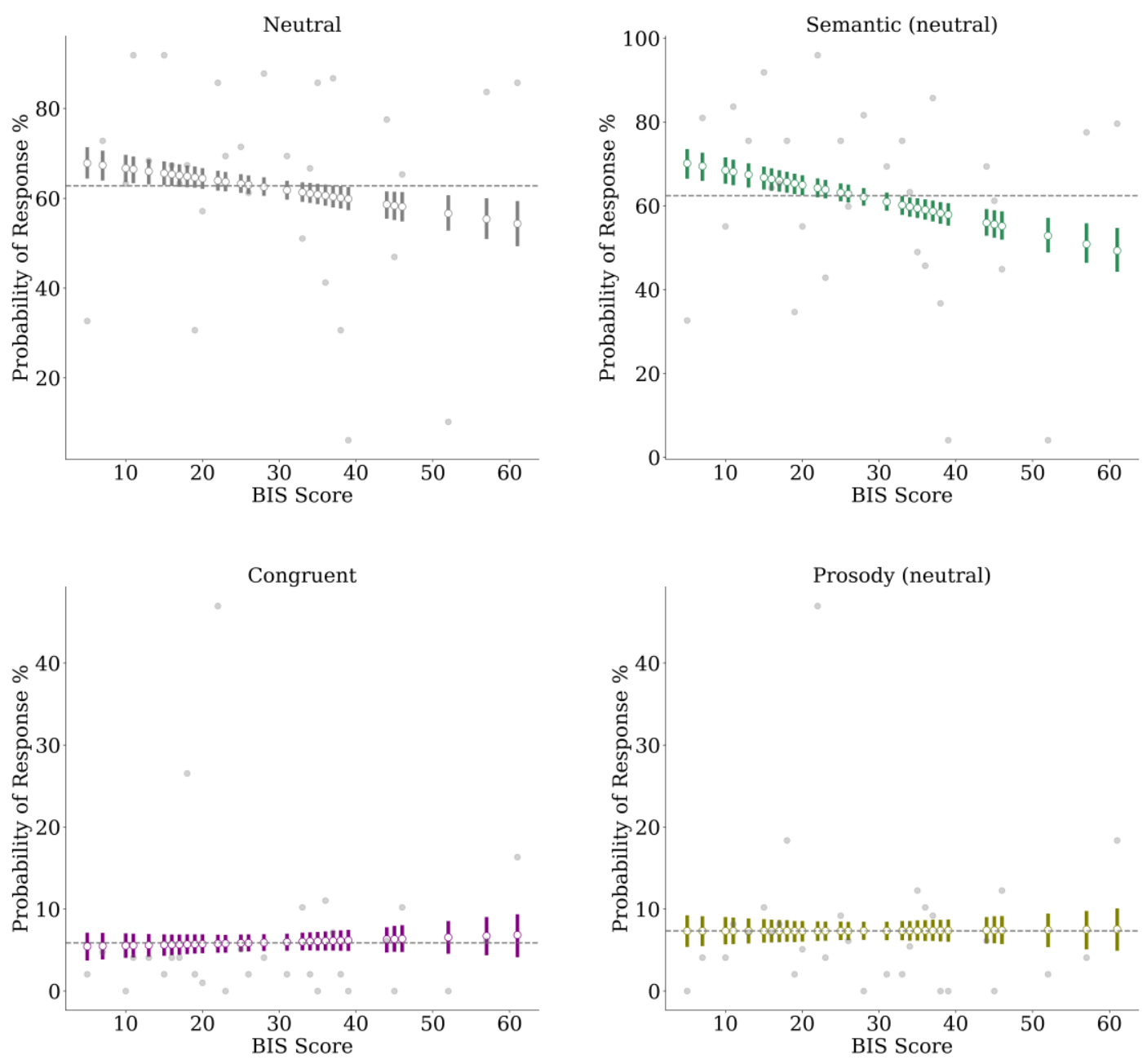

Figure 10. Experiment 4 accuracy estimates. Plots show responses to stimuli containing neutral semantics. Circles represent posterior means; bars indicate highest density intervals (HDIs). Grey dots indicate raw means by BIS score. Grey dashed lines indicate estimated posterior median accuracy by median BIS per condition.

Robust regression results indicate that as BIS increases RTs remain generally constant. RTs to neutral Semantic remain at around $1650 \mathrm{~ms}$ and RTs to Neutral at around $1770 \mathrm{~ms}$. This indicates that responses to neutral Semantic are around $120 \mathrm{~ms}$ slower than responses to Neutral. Note that estimations indicate precise HDIs not close to overlap. Although a robust regression was performed to account for outliers, this extreme RT constancy needs to be interpreted cautiously. See Table 10 and Figure 11 for summaries of these results. 
Table 10. Experiment 4 reaction time estimates

\begin{tabular}{cccccc}
\hline BIS & Sentence Type & Mean & SD & HDI 5\% & HDI 95\% \\
\hline 5 & Neutral & 1648.93 & 13.21 & 1626.01 & 1669.27 \\
\hline 5 & Semantic (neutral) & 1770.88 & 13.11 & 1749.85 & 1792.94 \\
\hline 62 & Neutral & 1655.56 & 18.41 & 1624.90 & 1685.88 \\
\hline 62 & Semantic (neutral) & 1776.20 & 18.86 & 1745.15 & 1807.48 \\
\hline
\end{tabular}

Note. Estimates are reaction times (in milliseconds) from expectation's posterior (intercept plus slope).

Neutral Semantic Regressions
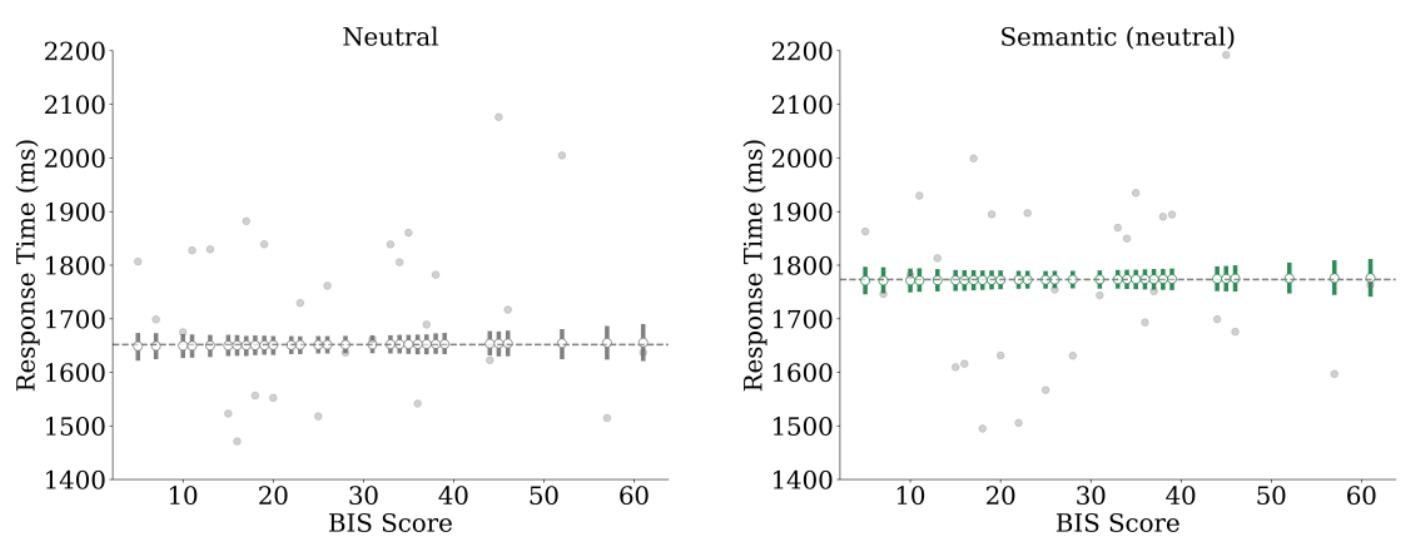

Figure 11. Experiment 4 reaction time estimates. Plots show responses to stimuli containing neutral semantics. Circles represent posterior means; bars indicate highest density intervals (HDIs). Grey dots indicate raw means by BIS score. Grey dashed lines indicate estimated posterior median accuracy by median BIS per condition.

In summary, Experiment 4 results indicate that accuracy is similarly moderately low for both Neutral and neutral Semantic sentence types, with medians around 60\%. Estimated accuracy shows a moderate decrease as a function of BIS for both types, suggesting relatively relevant effects of BIS. Median RT reaches nearly $1700 \mathrm{~ms}$ for Neutral and nearly 1750ms for neutral Semantic, indicating a near 50ms (but estimated to be around $100 \mathrm{~ms}$ ) slow-down for responses to neutral Semantic. However, neither of the two types shows changes as a function of BIS, suggesting no BIS effects. 


\section{General Discussion}

Results indicate that Experiment 1, where participants answered to threatening prosody (Prosody and Congruent), shows a small RT increase s a function of BIS for Congruent stimuli, but small to negligible changes for Prosody. However, we did not observe the predicted increase in accuracy as BIS increased, instead finding a small tendency in the opposite direction for Congruent. Moreover, the task-irrelevant cue (semantic threat) affected processing. BIS effects were observed with most certainty when this cue was congruent with prosody; its effect can also be observed in the slightly increased false alarm rate in the Semantic condition compared to the Neutral condition. A very different pattern of results was observed in Experiment 2, where participants answered to threatening semantics (Semantic and Congruent), we observed strong effects of BIS for both RT and accuracy, but again not fully consistent with our predictions. For accuracy we found that increased BIS was associated with better performance, also showing a similar small effect of the irrelevant cue (Prosody). However, increased BIS was associated with substantially faster responses in this task. These robust effects of BIS were observed for Congruent stimuli and Semantic stimuli alike, although the latter were somewhat more difficult as indicated by their overall slower responses. So far, we see a pattern of results consistent with a role of anxiety in threatening speech processing, but which is not consistent with our hypotheses, in which response inhibition associated with anxiety should leading to higher accuracy at the cost of slower response times (especially as the task length or interval for response becomes longer).

When we consider Experiments 1 and 2 together, our findings do not align well with the hypothesis that increased anxiety leads to RT increase (speed slow-down) when stimuli are longer and more complex, and processing is more difficult (see Cisler and Koster, 2010). And it certainly does not align with our hypothesis that semantic and prosodic threat should affect anxious participants in comparable ways, strong BIS effects were evident only when participants attended and responded to semantic threat. While the present findings do not allow us to unambiguously attribute these differences directly to semantic and prosodic speech informational properties, their effects in the present experimental context may allow some cautious interpretation. In particular, we note that the prosodic task was clearly easier than the semantic task, with much higher accuracy and response times. Due to the nature of the stimuli and the reliable discriminability of the acoustic cues distinguishing threatening from neutral prosody, it could be possible to 
respond very early indeed, before the full semantic content is available. If so, some aspects of Experiment 1 might be more comparable to those studies in the literature where short and intense stimuli are employed, and effects associated with over-attention to threat might be expected (see Cisler and Koster, 2010). Instead, participants responded more slowly than purely attending to acoustic cues would require, and there was some evidence that the semantic cue affected processing to some extent despite its task-irrelevance. Nonetheless we found effects of BIS despite this variability. On the other hand, the semantic task in Experiment 2 exhibited strong effects of BIS; this task was more difficult and required longer attention to the stimuli before accurate responses were possible, yet anxious people were both faster and more accurate than less anxious people.

Before moving on to relate these findings more broadly to the literature, it is important to consider the results of the control experiments, as responses to neutral stimuli can reveal whether the observed effects of anxiety occur only when the stimuli depict threat in the dimension of interest; if they occur for stimuli depicting threat in any way; or if they are simply a product of the task rather than the specific stimuli presented on a given trial. First, in Experiment 3 (respond to prosodically neutral stimuli), we found strong and reliable effects of BIS on both accuracy and RTs: anxious people were faster and more accurate than less anxious people. This is in strong contrast to Experiment 1 in which weak effects of BIS, tending in the opposite direction, were observed. This may indicate that more anxious participants are better at diverting attention from threat (focusing on neutral stimuli), in particular for longer stimuli (e.g. Koster et al., 2006). So, they find it less effortful to complete Experiment 3 than Experiment 1, as in the latter the required focus on threat may have induced delayed disengagement from threat (Cisler and Koster, 2010). Nevertheless, it may also be the case that less inhibited participants (lower BIS) are worse at focusing on less attractive stimuli (i.e. neutral), as opposed to, for instance, happy stimuli (e.g. Robinson et al., 2011). Although a combination of both options is possible, the present study does not allow us to distinguish them. What is possible to say, however, is that stimuli do have a role to play when it comes to response variation, and present results are not purely task-dependent. Finally, in Experiment 4, where participants answered to neutral semantics (Prosody and Neutral), shows a decrease in accuracy and no changes in RT as a function of BIS. Again, this does not align 
with our hypotheses, and indicates an opposite pattern respect to previous findings in the go/no-go paradigm (i.e. Grillon et al., 2017).

Taken in combination, we find a complex pattern of results, overall inconsistent with a simple explanation in which different facets or aspects of threatening speech affect anxious people in the same ways, neither consistent with the hypothesis of a speed-up of responses but loss of accuracy when stimuli are faster/shorter or tasks induce pressure. Contrary to previous perspectives (Bar-Haim et al., 2007; Cisler and Koster, 2010), Experiment 1 and 2 fail to induce strong delayed disengagement from threat. In particular, Experiment 2 and 3 show strong threat aversion effects, not supposed to be present for stimuli longer than 1500ms (Cisler and Koster, 2010). Although, it is important to emphasise that the threatening lexical items could appear at any position within the sentence, functioning as potentially de facto short sudden stimuli (unless easily predictable from sentence context); which may explain their threat aversion (overattention) effects. Even so, these results are conflicting, and indicate that the present tasks may not be sufficient to disentangle the difference between fast responses or delayed responses. That is, present stimuli may be insufficiently long /short, and/or the task may be insufficiently hard/easy, to induce a response coherent with previous theories. Nevertheless, we found overall reliable associations between anxiety and threatening speech processing, that also appear to differ between speech informational properties (i.e. prosody and semantics), a problem rarely addressed by previous literature (for an exception and exploratory approach see: Pell et al., 2015).

When we consider the present effects of Prosody, it is possible that the effects we observed are not speech-specific, as previous research has observed a similar slow-down of RTs for more anxious people and no evidence of accuracy effects in a task using aversive non-language-related sounds (Wang et al., 2019). Previous research on attentional switch from threatening stimuli to a response cue has also shown overengagement with threat patterns (Fox et al., 2002; Massar et al., 2011). Recent research on induced anxiety has observed that participants answering to faces in a go/no-go task tend to slow down their responses when under threat of electrical shock (Aylward et al., 2017). This was irrespective of stimuli emotional congruency, and had no effects on accuracy. In other words, participants in the anxiety condition were more cautious with their responses, in particular because of fearful faces capturing their attention, resulting in slowing down responses to achieve better accuracy. Even so, present results indicate 
that changing the target from threatening to neutral prosody (going from Experiment 1 to Experiment 3) drastically improves the responses of participants with higher BIS levels. This may be due, however, to the fact that their focus may be shifting from prosody to semantics. In other words, more anxious participants may have used the strategy to focus on Semantic (semantic threat) to find neutral prosody sentences (as in Experiment 2). After all, the neutral-Prosody condition corresponds to Semantic stimuli. However, this does not explain the effect of Neutral stimuli, which are both semantically and prosodically neutral.

When participants answer to threatening semantics (Experiment 2), there is no accuracy-speed trade-off either. As anxiety (BIS) increases participants become faster and more accurate (similar to Experiment 3). This occurs irrespective of stimulus type, though responses to Congruent are generally faster. This may simply indicate that participants used the strategy of focusing on the prosodic threat of Congruent sentences. In other words, participants have two cues (semantic and prosody), which may facilitate earlier recognition of threat. When participants answered to neutral semantics (Experiment 4), they also answered faster to Neutral than to neutral-Semantic stimuli, but no effect of BIS was observed. However, accuracy did show a similar decrease for both sentence types. This may indicate that more anxious participants attempted to speed-up their responses by sacrificing accuracy and they simply reached similar levels as less anxious participants. This could be interpreted as a speed-accuracy trade-off of sorts. Nevertheless, a clearer interpretation could be that threat aversion to semantic threat is greater for anxious people. Increased threat avoidance (Koster et al., 2005), inducing over-attention to threat (e.g. Bar-Haim et al., 2007), would explain why more anxious participants manage to 'catch up' with less anxious participants. And, impaired inhibitory control (Robinson et al., 2019) could explain why this attempt of speeding-up would also result in lower accuracy. This goes in line with the idea of lexical items having more explicit harmful meanings than sentences' prosody, so Semantic stimuli would be more expressive of potential threat. Nevertheless, this pattern of results is not sufficiently clear to assert this interpretation, in particular because it is not consistent with Experiment 3's threat-aversion interpretation. It is also possible that accuracy results are affected by outliers or another type of artefact, indicating that bigger sample size and replication of present results may be required. Even so, these results indicate, again, that effects are not purely task-dependent. 
Notwithstanding, present results are not sufficient to provide evidence for effects of anxiety on speech processing given multistep models of language (Kotz and Paulmann, 2011) and anxiety (Bar-Haim et al., 2007). As discussed above, the general pattern of results indicates that stimuli may not be the most adequate to test phasic effects, given that these happen in much shorter durations. We initially expected that task pressure would be enough to account for this. However, the high variability of sentences, in particular of lexical items within Semantic sentences, greatly limits this possibility. Other important caveat of present study is that additional control conditions may be required. Although diverting the task requirement from targeting threatening sentences to targeting neutral sentences in Experiments 3 and 4 provides a degree of control, this still implies that participants are exposed to threat. Hence, future experiments should directly address the difference between threatening and neutral stimuli, such as comparing Congruent versus Neutral stimuli in different tasks. Present sample sizes may also be a limitation, although present estimates also show relatively good precision, the fact that participant varying intercepts could not be excluded in models due to convergence issues may indicate that more information is required. High variation in participant responses may also induce a distortion in the estimated effects, possibly accounting for the high (though still coherent) variability of effects across experiments. Furthermore, this may be a problem that extends beyond this paper to the literature in general, as the inconsistency in present results resembles that of the literature. For instance, metanalyses on social anxiety (Chen et al., 2020) or attention bias (Beard et al., 2012) have shown that variation in threatening stimuli modality can induce different and seemingly inconsistent effects. Similarly, present results indicate that variations in information types (i.e. prosody and semantic), even within the speech modality, can also alter response patterns, in particular as trait anxiety increases.

In conclusion, present results indicate noticeable effects of BIS (trait anxiety) on language processing, in terms of both stimuli type and task. While responses to prosodic threat are compatible with over-engagement with threat, responses to semantic threat are compatible with over-attention to threat. This may indicate that theoretical models cannot properly account for stimuli informational variation, or that our stimuli are not suitable to appropriately test models' assumptions. Control experiments, where participants answered to the neutral counterparts of stimuli, suggest a different explanation. While answers to neutral prosody are compatible with models proposing 
increased aversion to threat for longer stimuli, answers to neutral semantics seem to suggest threat avoidance effects. Hence, further research is necessary to establish whether responses in the present paradigm are consistent across experiments and what interpretation or models can be better supported by the evidence they will provide.

\section{Declarations}

\section{Conflict of interests}

None.

Data and Code Availability

All data, analyses' scripts, and additional info can be found at the Open Science Framework (OSF) repository: https://osf.io/ptcr9/

\section{Authors Contributions}

Contributions to the study were as follows. Busch-Moreno: Conceptualization, Methodology, Software, Analysis, Investigation, Resources, Curation, Writing, Visualization, Administration. Vinson: Conceptualization, Methodology, Resources, Revision, Supervision, Administration, Writing. Smekal: Materials, Curation. Gorgol, Li, Ng, and Zhang: Data collection, Investigation, Curation. Xi: Implementation, Data collection, Curation, Revision.

\section{Funding}

This research is supported by CONICYT Becas Chile 72170145

(https://www.conicyt.cl/).

\section{Ethical Approval}

This research was approved by University College London's Ethics Committee. Approval code: ID EP-2017-003.

Consent for Publication

All authors gave consent before submission. 


\section{Consent to Participate}

All authors and participants gave their consent for taking part in this study.

\section{References}

Aylward, J., Valton, V., Goer, F., Mkrtchian, A., Lally, N., Peters, S., Limbachya, T., \& Robinson, O. J. (2017). The impact of induced anxiety on affective response inhibition. Royal Society Open Science, 4(6), 170084. https://doi.org/10.1098/rsos.170084

Bar-Haim, Y., Lamy, D., Pergamin, L., Bakermans-Kranenburg, M. J., \& van IJzendoorn, M. H. (2007). Threat-related attentional bias in anxious and nonanxious individuals: A meta-analytic study. Psychological Bulletin, 133(1), 1-24. https://doi.org/10.1037/0033-2909.133.1.1

Beard, C., Sawyer, A. T., \& Hofmann, S. G. (2012). Efficacy of Attention Bias Modification Using Threat and Appetitive Stimuli: A Meta-Analytic Review. Behavior Therapy, 43(4), 724-740. https://doi.org/10.1016/j.beth.2012.01.002

Chen, J., Short, M., \& Kemps, E. (2020). Interpretation bias in social anxiety: A systematic review and meta-analysis. Journal of Affective Disorders, 276, 1119-1130. https://doi.org/10.1016/j.jad.2020.07.121

Cisler, J. M., \& Koster, E. H. W. (2010). Mechanisms of attentional biases towards threat in anxiety disorders: An integrative review. Clinical Psychology Review, 30(2), 203-216. https://doi.org/10.1016/j.cpr.2009.11.003

Corr, P. J., \& Cooper, A. J. (2016). The Reinforcement Sensitivity Theory of Personality Questionnaire (RST-PQ): Development and validation. Psychological Assessment, 28(11), 1427-1440. https://doi.org/10.1037/pas0000273

Dymond, S., Dunsmoor, J. E., Vervliet, B., Roche, B., \& Hermans, D. (2015). Fear Generalization in Humans: Systematic Review and Implications for Anxiety Disorder Research. Behavior Therapy, 46(5), 561-582. https://doi.org/10.1016/j.beth.2014.10.001 
Fox, E., Russo, R., \& Dutton, K. (2002). Attentional bias for threat: Evidence for delayed disengagement from emotional faces. Cognition \& Emotion, 16(3), 355-379. https://doi.org/10.1080/02699930143000527

Gray, J. A., \& McNaughton, N. (2000). The neuropsychology of anxiety: an enquiry into the functions of the septo-hippocampal system, second edition (Second). Oxford University Press.

Grillon, C., Robinson, O. J., Krimsky, M., O’Connell, K., Alvarez, G., \& Ernst, M. (2017). Anxiety-mediated facilitation of behavioral inhibition: Threat processing and defensive reactivity during a go/no-go task. Emotion, 17(2), 259-266. https://doi.org/10.1037/emo0000214

Grimshaw, G. M., Kwasny, K. M., Covell, E., \& Johnson, R. A. (2003). The dynamic nature of language lateralization: effects of lexical and prosodic factors. Neuropsychologia, 41(8), 1008-1019. https://doi.org/10.1016/s00283932(02)00315-9

Heller, W., Nitschke, J. B., Etienne, M. A., \& Miller, G. A. (1997). Patterns of regional brain activity differentiate types of anxiety. Journal of Abnormal Psychology, 106(3), 376-385. https://doi.org/10.1037/0021-843x.106.3.376

Jadoul, Y., Thompson, B., \& de Boer, B. (2018). Introducing Parselmouth: A Python interface to Praat. Journal of Phonetics, 71, 1-15. https://doi.org/10.1016/j.wocn.2018.07.001

Koster, E. H. W., Crombez, G., Verschuere, B., \& De Houwer, J. (2006). Attention to Threat in Anxiety-prone Individuals: Mechanisms Underlying Attentional Bias. Cognitive Therapy and Research, 30(5), 635-643. https://doi.org/10.1007/s10608-0069042-9

Koster, E. H. W., Verschuere, B., Crombez, G., \& Van Damme, S. (2005). Time-course of attention for threatening pictures in high and low trait anxiety. Behaviour Research and Therapy, 43(8), 1087-1098. https://doi.org/10.1016/j.brat.2004.08.004

Kotz, S. A., \& Paulmann, S. (2011). Emotion, Language, and the Brain. Language and Linguistics Compass, 5(3), 108-125. https://doi.org/10.1111/j.1749818x.2010.00267.x

Kruschke, J. K. (2013). Bayesian estimation supersedes the t test. Journal of Experimental Psychology: General, 142(2), 573-603. 
https://doi.org/10.1037/a0029146

Massar, S. A. A., Mol, N. M., Kenemans, J. L., \& Baas, J. M. P. (2011). Attentional bias in high- and low-anxious individuals: Evidence for threat-induced effects on engagement and disengagement. Cognition \& Emotion, 25(5), 805-817. https://doi.org/10.1080/02699931.2010.515065

McNaughton, N., \& Corr, P. J. (2004). A two-dimensional neuropsychology of defense: fear/anxiety and defensive distance. Neuroscience \& Biobehavioral Reviews, 28(3), 285-305. https://doi.org/10.1016/j.neubiorev.2004.03.005

McNaughton, N., \& Gray, J. A. (2000). Anxiolytic action on the behavioural inhibition system implies multiple types of arousal contribute to anxiety. Journal of Affective Disorders, 61(3), 161-176. https://doi.org/10.1016/s01650327(00)00344-x

Paulmann, S., \& Kotz, S. A. (2008). An ERP investigation on the temporal dynamics of emotional prosody and emotional semantics in pseudo- and lexical-sentence context. Brain and Language, 105(1), 59-69. https://doi.org/10.1016/j.bandl.2007.11.005

Pell, M. D., Rothermich, K., Liu, P., Paulmann, S., Sethi, S., \& Rigoulot, S. (2015).

Preferential decoding of emotion from human non-linguistic vocalizations versus speech prosody. Biological Psychology, 111, 14-25. https://doi.org/10.1016/j.biopsycho.2015.08.008

Robinson, O. J., Letkiewicz, A. M., Overstreet, C., Ernst, M., \& Grillon, C. (2011). The effect of induced anxiety on cognition: threat of shock enhances aversive processing in healthy individuals. Cognitive, Affective, \& Behavioral Neuroscience, 11(2), 217227. https://doi.org/10.3758/s13415-011-0030-5

Robinson, O. J., Krimsky, M., \& Grillon, C. (2013). The impact of induced anxiety on response inhibition. Frontiers in Human Neuroscience, 7. https://doi.org/10.3389/fnhum.2013.00069

Robinson, O. J., Pike, A. C., Cornwell, B., \& Grillon, C. (2019). The translational neural circuitry of anxiety. Journal of Neurology, Neurosurgery \& Psychiatry, 90, 13531360. https://doi.org/10.1136/jnnp-2019-321400

Salvatier, J., Wiecki, T. V., \& Fonnesbeck, C. (2016). Probabilistic programming in Python using PyMC3. PeerJ Computer Science, 2, e55. https://doi.org/10.7717/peerjcs.55 
Spielberg, J. M., De Leon, A. A., Bredemeier, K., Heller, W., Engels, A. S., Warren, S. L., Crocker, L. D., Sutton, B. P., \& Miller, G. A. (2013). Anxiety type modulates immediate versus delayed engagement of attention-related brain regions. Brain and Behavior, 3(5), 532-551. https://doi.org/10.1002/brb3.157

Wambacq, I. J. A., \& Jerger, J. F. (2004). Processing of affective prosody and lexicalsemantics in spoken utterances as differentiated by event-related potentials. Cognitive Brain Research, 20(3), 427-437. https://doi.org/10.1016/j.cogbrainres.2004.03.015

Wang, Y., Xiao, R., Luo, C., \& Yang, L. (2019). Attentional disengagement from negative natural sounds for high-anxious individuals. Anxiety, Stress, \& Coping, 32(3), 298311. https://doi.org/10.1080/10615806.2019.1583539

Warriner, A. B., Kuperman, V., \& Brysbaert, M. (2013). Norms of valence, arousal, and dominance for 13,915 English lemmas. Behavior Research Methods, 45(4), 11911207. https://doi.org/10.3758/s13428-012-0314-x 